\title{
Nuclear Lattices, Mass and Stability
}

\author{
Henry Gu Cao, Zhiliang Cao, Wenan Qiang \\ Northwestern University, Evanston, USA \\ Email: henrycao2018@u.northwestern.edu
}

Received 20 April 2015; accepted 7 May 2015; published 13 May 2015

Copyright (C) 2015 by authors and OALib.

This work is licensed under the Creative Commons Attribution International License (CC BY).

http://creativecommons.org/licenses/by/4.0/

\section{(c) (i) Open Access}

\section{Abstract}

A nucleus has a lattice configuration, a mass, and a half-life. There are many nuclear theories: BCS formalism focuses on Neutron-proton (np) pairing; AB initio calculation uses NCFC model; SEMF uses water drop model. However, the accepted theories give neither précised lattices of lower mass nuclei, nor an accurate calculation of nuclear mass. This paper uses the results of the latest Unified Field Theory (UFT) to derive a lattice configuration for each isotope. We found that a simplified BCS formalism can be used to calculate energies of the predicted lattice structure. Furthermore, mass calculation results and NMR data can be used to determine the right lattice structure. Our results demonstrate the inseparable relationship among nuclear lattices, mass, and stability. We anticipate that our essay will provide a new method that can predict the lattice of each isotope without the use of advanced mathematics. For example, the lattice of an unknown nucleus can be predicted using trial and error. The mass of the nuclear lattice can be calculated. If the calculation result matches the experimental data and NMR pattern supports the lattice as well, then the predicted nuclear lattice configuration is valid.

\section{Keywords}

Neutron-Proton Pairing, Particle-Number Projection, Nuclear Lattice, Particle Physics, BCS

Subject Areas: Nuclear Physics, Particle Physics, Theoretical Physics

\section{Introduction}

BCS theory was proposed by John Bardeen, Leon Cooper, and John Robert Schrieffer (BCS) in 1957; they received the Nobel Prize in Physics for this theory in 1972. As an important nuclear theory, BCS [1]-[9] has made many important predictions. According to BCS formalism, the BCS ground state can be represented as:

$$
|\Psi\rangle=\prod_{j>0}\left|\Psi_{j}\right\rangle
$$

Neutron-Proton (np) pairing effects play an important role in BCS theory. This paper does not consider np 
pairing; it only considers the nuclear lattice.

Unified Field Theory (UFT) [10]-[21] predicted the structures [10]-[13] of the proton and the neutron. According to UFT, a nucleon has three axes and a few other lower energy components. Each component plays different roles in nuclear stability. Two of three axes of a nucleon have $98 \%$ of the energy and they give nuclei the ability to strongly interact with one another and line up nucleons as a lattice. The lower energy axis for the proton is charged and for a neutron, in a heavy nucleus, is neutral. The weak interactions between lower energy axes line up nucleons along the third direction [10].

Due to wave resonance, there are only a few configurations available for the lower energy components as they reach harmonic state over time. The massive axes [10]-[14], roaming waves, and residential waves are weakly/ strongly interacting at bonding points and impacting lattice feature sites.

When an interaction in the nucleus reaches resonance state, the wave formation can be expressed as simplified BCS equation:

$$
E=\prod_{1}^{n} S_{j}
$$

$\mathrm{Si}$ is an energy level in an electron mass unit or wave formation parameters. $2 * \mathrm{Si}$ is an integer.

UFT consider the electron mass the quantum unit of particle/energy in nuclei as other energies resonant with charged energy. In the data book, the nuclear masses are in atomic mass unit. To convert it to an electronic mass unit:

$1 \mathrm{u}=1822.8884845 \mathrm{e}$

It is difficult to get accurate atomic mass [22]. Using atomic mass in electronic mass unit is a novel technique deeply rooted in UFT similar to other concepts from UFT. Many recent physics papers [23]-[31] are studying similar issues.

This paper uses nuclear mass data and NMR results (Table 1) as experimental foundations.

\subsection{UFT Concepts}

The main structure of the Proton and Neutron are their axes [10]-[14]: $\mathrm{A}^{2}, \mathrm{~A}^{2}$ and $\mathrm{A}$ (some time B).

The component " $A$ " has the following mass formula from (1) in unit of electron mass:

$\mathrm{A}=2 * 3 * 5$ (charged)

In addition,

$\mathrm{A}^{2}=(2 * 3 * 5) *(2 * 3 * 5)$

Component " $\mathrm{B}$ " has the following mass formula:

$\mathrm{B}=2 * 2 * 4$ (charged) or $2 * 2 * 2 * 2$ (neutral)

Table 1. Masses for nuclei (experimental data).

\begin{tabular}{ccc}
\hline Isotope & Mass in Atomic Unit (u) & NMR Properties \\
\hline${ }^{1} \mathrm{H}$ & $1.007276466812(90)$ & Most Sensitive \\
${ }^{4} \mathrm{He}$ & 4.0015059 & Very Sensitive \\
${ }^{6} \mathrm{Li}$ & 6.0134768 & Low Sensitivity, Sharp Signal \\
${ }^{9} \mathrm{Be}$ & 9.0100138 & Low Sensitivity \\
${ }^{10} \mathrm{~B}$ & 10.010194 & Low Sensitivity \\
${ }^{12} \mathrm{C}$ & 11.996708 & Not NMR Active \\
${ }^{16} \mathrm{O}$ & 15.990525 & Not NMR Active \\
${ }^{25} \mathrm{Mg}$ & 24.979252 & Low Sensitivity \\
${ }^{27} \mathrm{Al}$ & 26.974405 & Very Sensitive \\
${ }^{28} \mathrm{Si}$ & 27.969245 & Not NMR Active \\
\hline
\end{tabular}




\subsection{Experimental and Theoretical Results}

The experimental data and theoretical data (Table 2) in chapter 3 matched closely as follow:

The nuclear mass is based on the following formula:

Atom mass $-\mathrm{Z} / 1822.8884845-(2 * \mathrm{Z}+8 * \mathrm{Z} / 4+\cdots) /(137 * 137 * 1822.8884845)$

${ }^{9} \mathrm{Be}$ and ${ }^{25} \mathrm{Mg}$ can be calculated with a small margin of error. In the other case, the calculation is more precise. Noticeably, a neutron is at the center for ${ }^{9} \mathrm{Be}$ and ${ }^{25} \mathrm{Mg}$ in predicted lattices and bonding energy is smaller, while the calculation formula is complex. ${ }^{10} \mathrm{~B}$ also has a larger margin of error due to its special lattice introduced unexplained strong interactions.

The precise calculation of ${ }^{27} \mathrm{Al}$ indicates that np paring is not an important factor in this theory. Even the worst result for ${ }^{25} \mathrm{Mg}$ above is better than the existing theories, such as Ab Initio calculation [32], and SEMF [33]. The precision of the above results demonstrates the merits of the new theory.

\subsection{Configuration of Proton}

If we use the electron mass as a unit, the mass of the proton (Figure 1) is:

$1836.15267(\mathrm{e})=2 \mathrm{~A}^{2}+\mathrm{A}+2 * 3+0.15267$

The structure formula is:

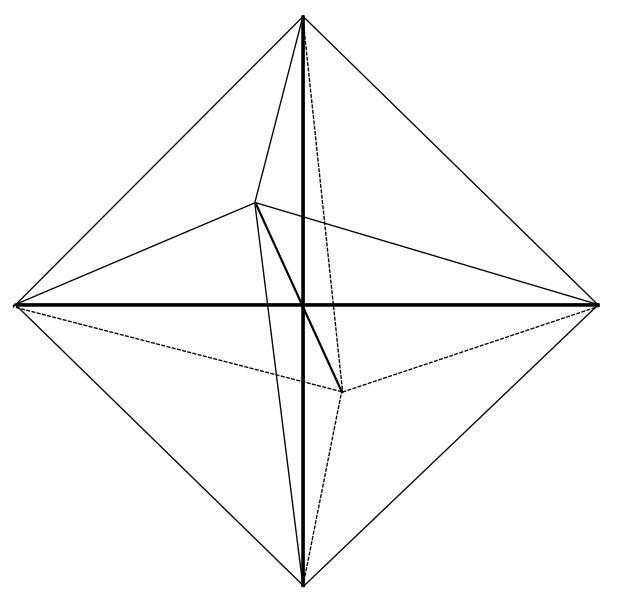

Figure 1. Proton.

Table 2. Comparing experimental data and theoretical data.

\begin{tabular}{ccc}
\hline Isotope & \multicolumn{2}{c}{ Mass in Atomic Unit (u) } \\
\cline { 2 - 3 } \\
${ }^{1} \mathrm{H}$ & Experimental & Theoretical \\
${ }^{4} \mathrm{He}$ & $1.007276466812(90)$ & 1.0072764660 \\
${ }^{6} \mathrm{Li}$ & 4.0015059 & $4.0015059(5)$ \\
${ }^{9} \mathrm{Be}$ & 6.0134768 & 6.0134767 \\
${ }^{10} \mathrm{~B}$ & 9.0100138 & 9.0100225 \\
${ }^{12} \mathrm{C}$ & 10.010194 & 10.010188 \\
${ }^{16} \mathrm{O}$ & 11.996708 & 11.996708 \\
${ }^{25} \mathrm{Mg}$ & 15.990525 & 15.990526 \\
${ }^{27} \mathrm{Al}$ & 24.979252 & 24.979246 \\
${ }^{28} \mathrm{Si}$ & 26.974405 & 26.974407 \\
\hline
\end{tabular}




\section{$2 \mathrm{~A}^{2}+\mathrm{A}+2 * 3$}

The additional mass 0.15267 is related to various interactions:

1. The structure $2 \mathrm{~A}^{2}$ strong interaction.

2. Weak interaction between charge and eight faces;

3. Weak interaction between $2 * 3$ and A.

\subsection{Configuration of Neutron}

Using the mass of electron as a unit mass, we derive the mass of a neutron to be:

$1838.68365987=2 \mathrm{~A}^{2}+\mathrm{A}+2 * 3+2.5+0.15267+0.030987$

The structure formula is:

$2 \mathrm{~A}^{2}+\mathrm{A}+2 * 3+2.5$

The additional mass 0.15267 can be interpreted the same as proton. Energy 0.030987 is result of the following interactions:

1. Strong interaction between 2.5 and two $\mathrm{A}^{2}$ structure;

2. Dissonant weak interaction between 2.5 and $2 * 3$;

3. Weak interaction between 2.5 and $2 * 3 * 5$;

\section{Results}

\subsection{Coupling of Proton and Neutron}

When a proton and a neutron form a nucleus (Figure 2):

1. One of axes $A^{2}$ from proton bonds with an $A^{2}$ axis from neutron;

2. Wave $2 * 3$ becomes $2 * 2$;

3. The wave 2.5 of neutron becomes 2 .

\subsection{Two Neutrons and One Proton}

Since there are two neutrons, two positive waves of energy level 3 are shared among them (Figure 3). A proton only contains three axes.

\subsection{Symmetrical Preferences}

Two pairs of interacting particles are arranged in the following manner:

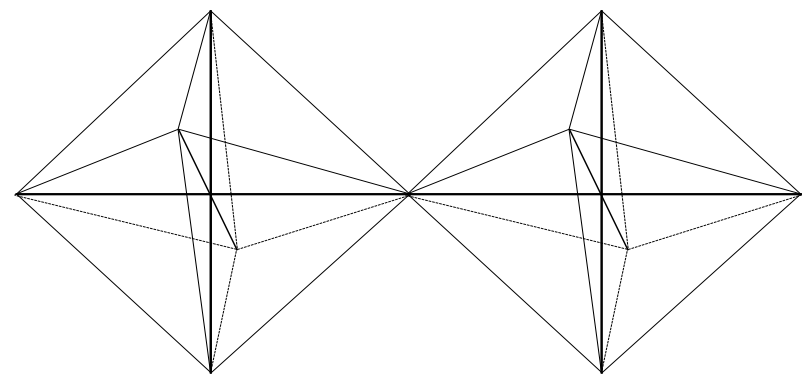

Figure 2. Coupling of proton and neutron.

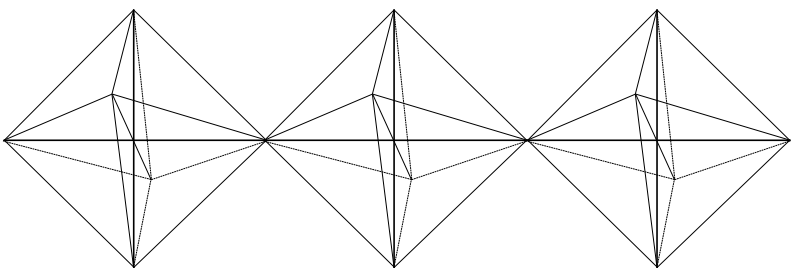

Figure 3. Two neutrons and one proton. 
The black squares (Figure 4) are neutrons. The gray squares are protons. In the above figure, protons P1 and P2 form a pair, N1 and N2 form a pair of neutrons. These types of pairings are pp/nn pairings. The pairings in single layered nuclei do not play important roles as many stabled nuclei have odd number of neutrons or protons.

${ }^{5} \mathrm{H}$ always decays to ${ }^{3} \mathrm{H}$ as follow according to experimental results (Figure 5).

The two additional neutrons in ${ }^{5} \mathrm{H}$ create complex energy wave pattern. ${ }^{3} \mathrm{H}$ has simpler preferred structure.

For the same reason, proton rich ${ }^{5} \mathrm{Be}$ has following decays confirmed by experimental results (Figure 6).

\subsection{Isotopes of Helium}

Helium (Figure 7) has two stable isotopes ${ }^{3} \mathrm{He}$ and ${ }^{4} \mathrm{He}$.

\subsection{Isotopes of Lithium}

Lithium (Figure 8) has two stable isotopes ${ }^{6} \mathrm{Li}$ and ${ }^{7} \mathrm{Li}$.

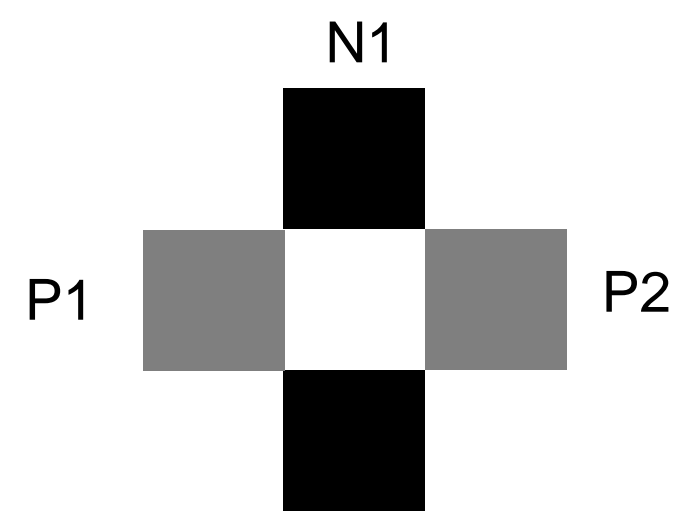

N2

Figure 4. Symmetrical lattice.

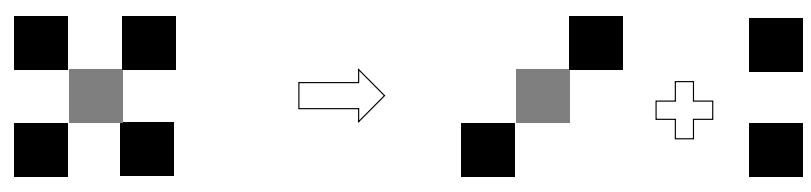

Figure $5 .{ }^{5} \mathrm{H}$ decay process.

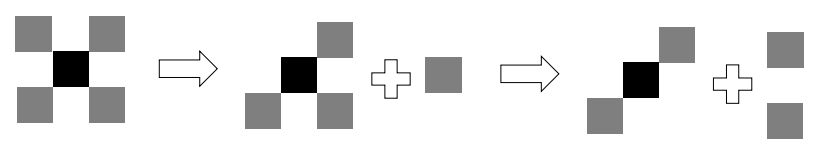

Figure $6 .{ }^{5} \mathrm{Be}$ decay process.

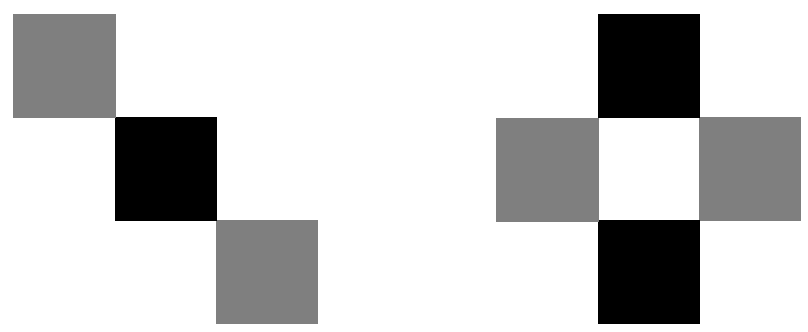

Figure 7. Isotopes of helium. 


\subsection{Isotopes of Beryllium}

Beryllium (Figure 9) has one stable isotope ${ }^{9} \mathrm{Be}$.

${ }^{8} \mathrm{Be}$ (Figure 10) has lower energy, but it can be decayed to stable structures.

${ }^{10} \mathrm{Be}$ is (Figure 11) pretty stable, but it still can decay.

\subsection{Isotopes of Boron}

Boron (Figure 12) has two stable isotopes ${ }^{10} \mathrm{~B}$ and ${ }^{11} \mathrm{~B}$.

\subsection{Isotopes of Carbon}

Carbon (Figure 13) has two stable isotopes and one relative stable isotope ${ }^{14} \mathrm{C}$.

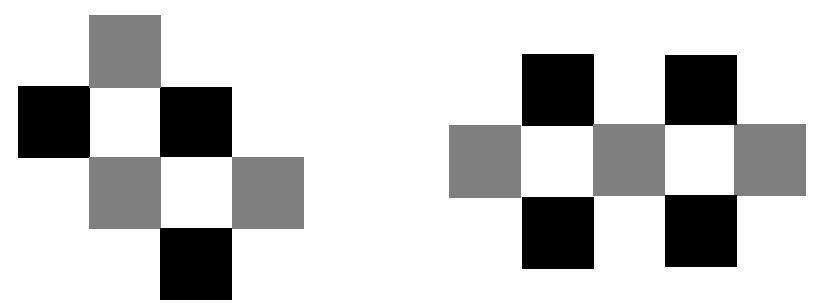

Figure 8. Isotopes of lithium.

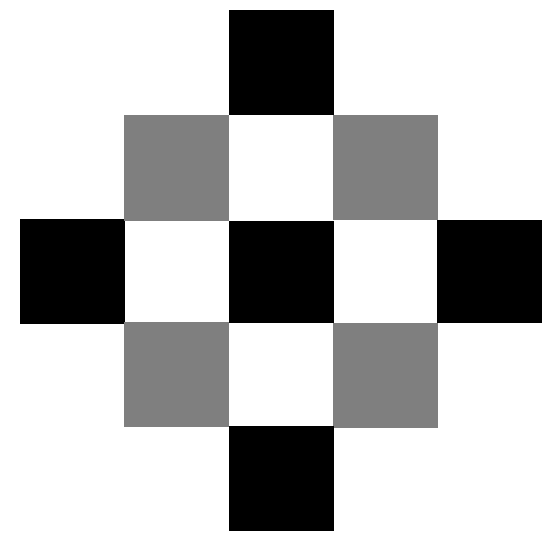

Figure 9. ${ }^{9} \mathrm{Be}$.

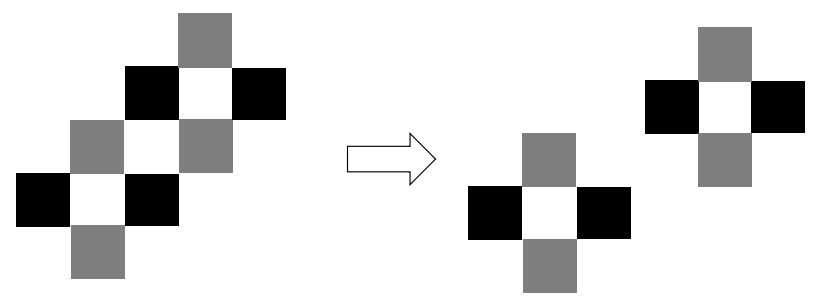

Figure $10 .{ }^{8} \mathrm{Be}$ decay process.

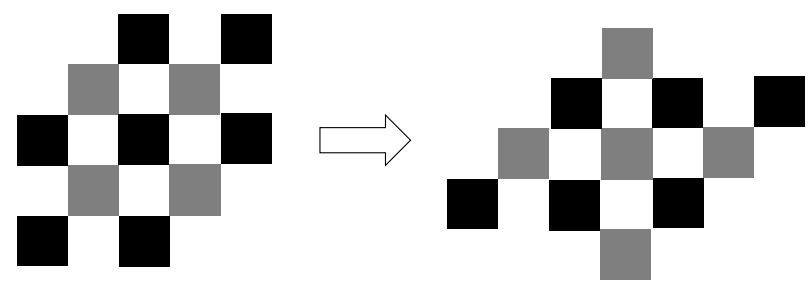

Figure $11 .{ }^{10}$ Be decay process. 
Only ${ }^{12} \mathrm{C}$ and ${ }^{13} \mathrm{C}$ are stable. ${ }^{14} \mathrm{C}$ (Figure 14) is less stable since it has a better alternative lattice ${ }^{14} \mathrm{~N}$.

\subsection{Isotopes of Nitrogen}

Nitrogen has two stable isotopes (Figure 15).

\subsection{Isotopes of Oxygen}

Oxygen has three stable isotopes (Figure 16).

\subsection{Isotopes of Fluorine}

Fluorine has only one stable isotope (Figure 17).
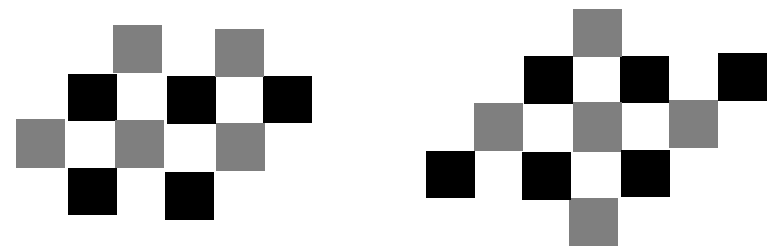

Figure 12. Isotopes of boron.
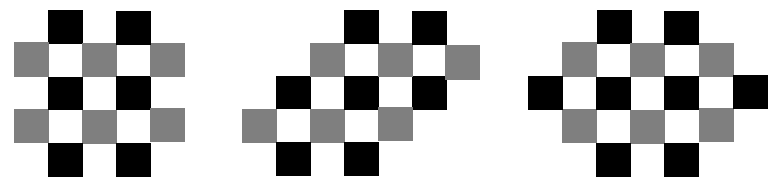

Figure 13. Isotopes of carbon.
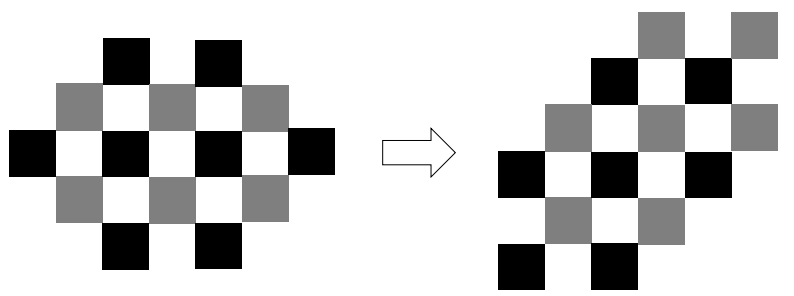

Figure $14 .{ }^{14} \mathrm{C}$ Decay process.
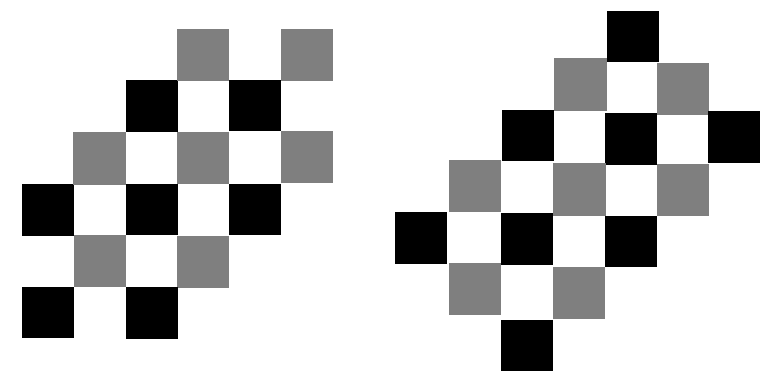

Figure 15. Isotopes of nitrogen.
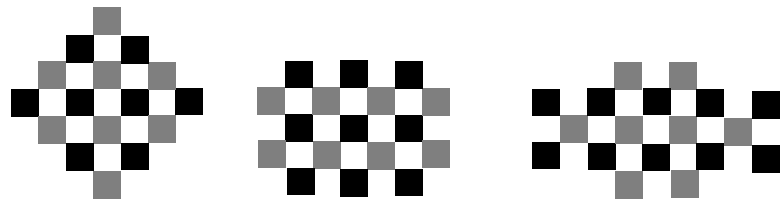

Figure 16. Isotopes of oxygen. 


\subsection{Isotopes of Neon}

Neon has three stable isotopes (Figure 18).

\subsection{Isotopes of Sodium}

Sodium has only one stable isotope (Figure 19).

\subsection{Isotopes of Magnesium}

Magnesium has three stable isotopes (Figure 20).

\subsection{Isotopes of Aluminium}

Aluminium has only one stable isotope (Figure 21).

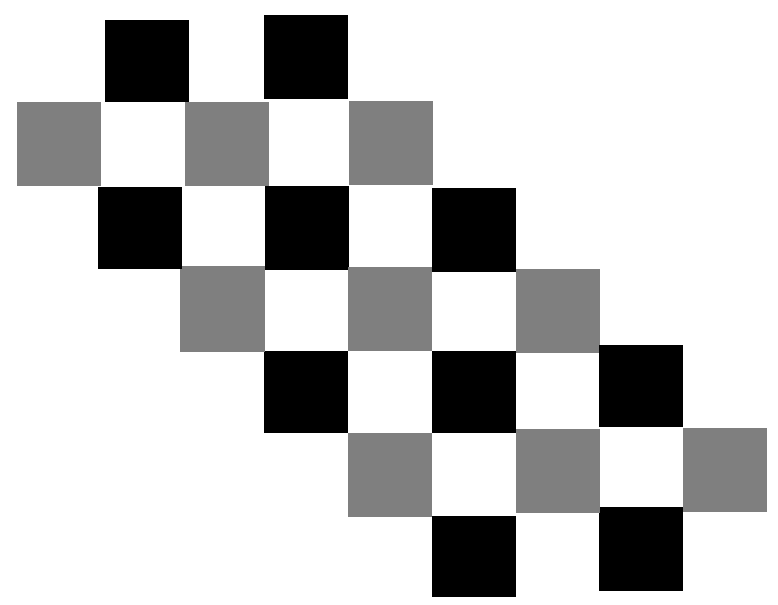

Figure 17. Isotopes of fluorine.
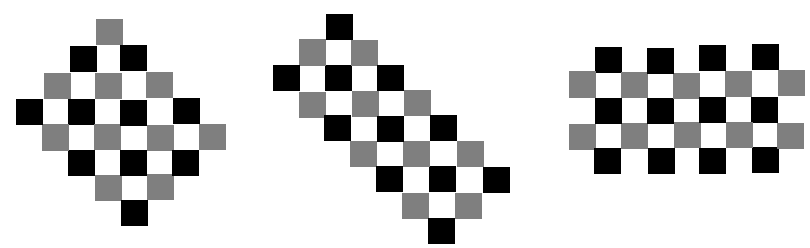

Figure 18. Isotopes of neon.

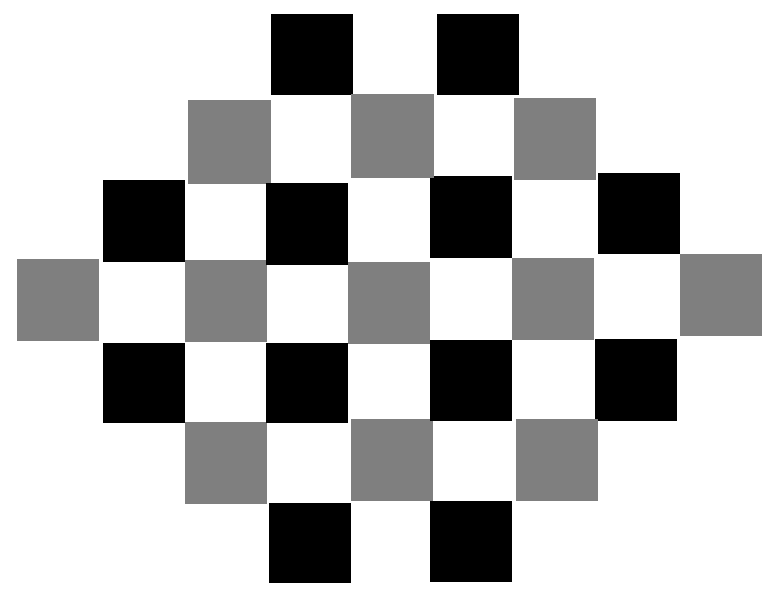

Figure 19. Isotopes of sodium. 


\subsection{Isotopes of Silicon}

Neon has three stable isotopes (Figure 22).

\subsection{Isotopes of Phosphorus}

Neon has only one stable isotope (Figure 23).

\section{Methods}

The paper uses trial and error to find the possible nuclear structure for any lower mass isotope by trying various layouts and choosing the best symmetrical layout. The selected layout has to be verified by mass calculation according to the wave interaction of the given lattice.

Even though strong interaction has a relatively long range compared to the size of the nucleus, the strong interaction of nucleons are through bonding points where the neighboring nucleons interact with each other via overlapping axes. The strong interaction energy is $137 \mathrm{e}$. Since the interactions are through axis $\mathrm{A}^{2}$ plus some additional energy, the strong interaction unit becomes:

$137 /\left(900+E_{0}\right)$
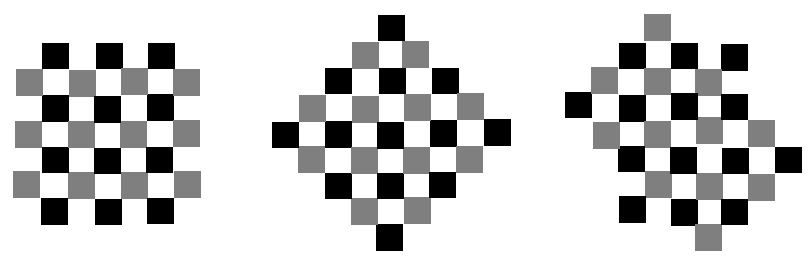

Figure 20. Isotopes of magnesium.

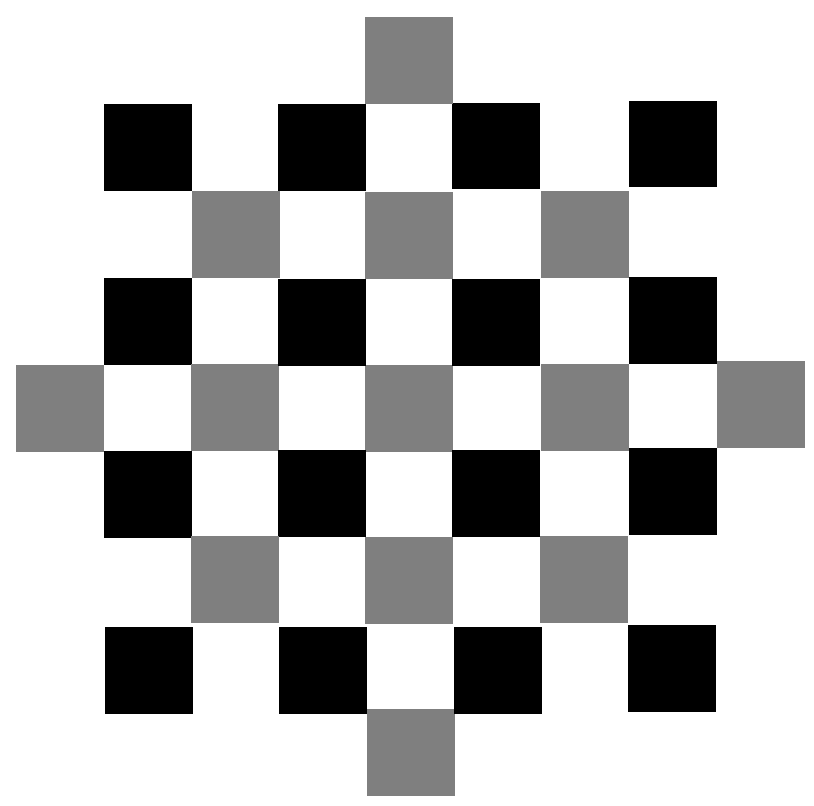

Figure 21. Isotopes of aluminium.
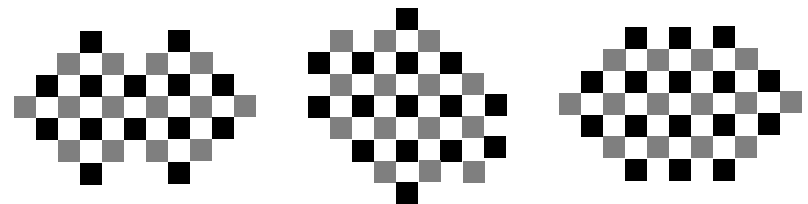

Figure 22. Isotopes of silicon. 


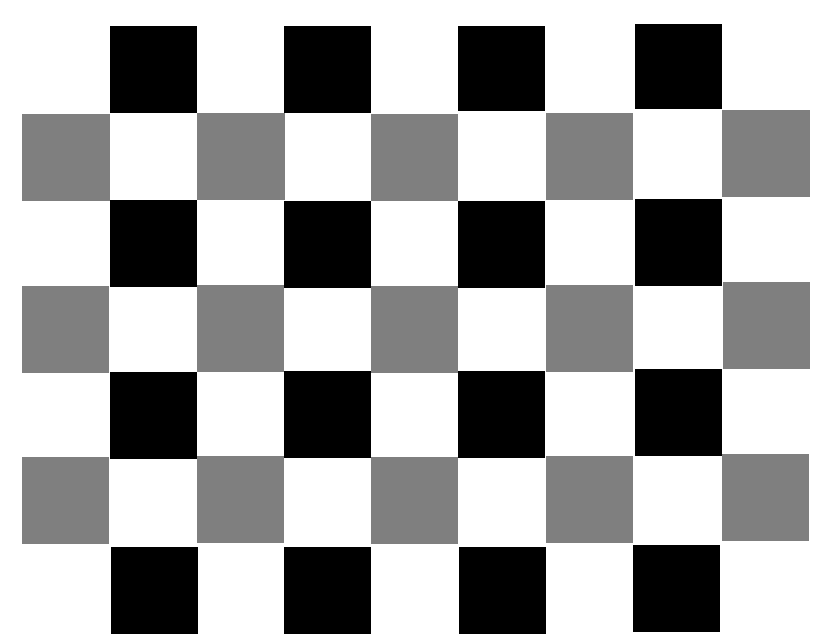

Figure 23. Isotopes of Phosphorus.

This interaction unit is distributed among the interaction pockets E, such as nucleon count and energy number. The bonding energy at the bonding point resonances is:

$137 /\left(\left(900+E_{0}\right) * E\right)$

From Formula (1):

$$
\frac{137}{\left(900+E_{0}\right) * \prod_{1}^{n} S_{j}}
$$

The weak interaction's unit is: $137 * 137$

When waves resonate with one another, the weak interaction energy is:

$$
\frac{1}{137 * 137 * \prod_{1}^{n} S_{i}}
$$

When waves are dissonant with one another, the compensate wave interaction factor is (can be strong or weak interaction):

$$
P / Q=\frac{\prod_{1}^{x} P_{i}}{\prod_{1}^{y} Q_{i}}
$$

The weak interaction energy becomes:

$$
\frac{P / Q}{137 * 137}
$$

The interaction energy represents the energy transporting speed per wave cycle. Since a higher energy wave has a higher frequency, it makes the transportation throughput at a bonding point constant.

There are many structural components in a nucleon. The energy level of each component in a nucleon exists mainly for the stability of the nucleus itself. In a nucleus, the components in the nucleons become similar. This can reduce the complexity of many body interactions in a nucleus.

Conversely, each nuclear configuration is unique. A generic formalism cannot replace the case-by-case study on each isotope. We calculate the mass of new isotopes in this chapter.

Strong interaction of the internal waves can be visually represented as a rope model (Figure 24).

$\mathrm{A}=2 * 3 * 5$ 


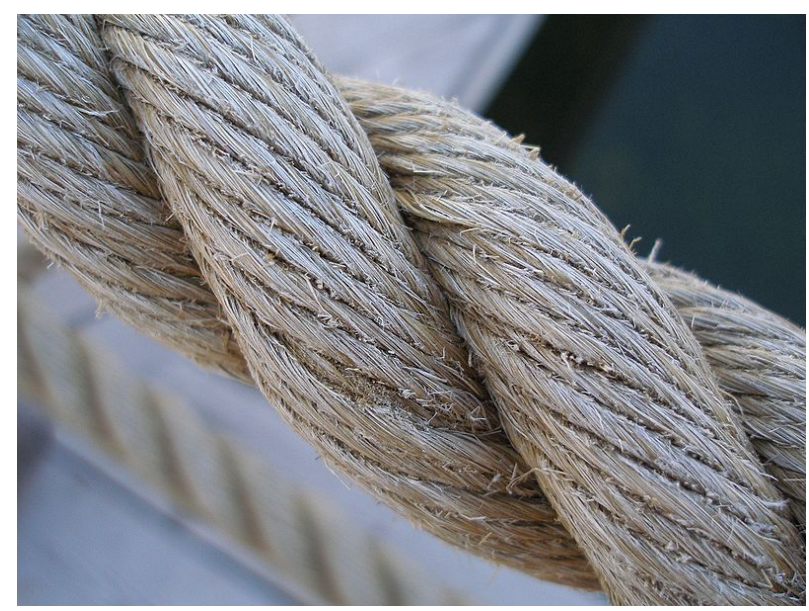

Figure 24. Strong interaction rope model.

The above formula is not just an equation; the formula shows how energy is formed like a rope (Figure 24). The inner wave is 2 (fine fiber of the rope), middle is 3 (fine string) and outer is 5 (the rope). The inner wave plus the middle wave $2+3=5$. The strong interaction multiplies and aggregates from the center to the outer part. Fibonacci series are physically represented as energy waves like $\mathrm{A}^{2}, \mathrm{~B}^{2}, \mathrm{~A}$, and $\mathrm{B}$, from central wave 2 to outer wave 5 (or 4 in case of B).

When wave $2 * 2$ interacts with $\mathrm{A}^{2}$, it interacts with the central waves of $\mathrm{A}^{2}$. Wave $2 * 3$ interacts with middle and outer part of wave $\mathrm{A}^{2}$.

The roles in the nucleon are similar to a transportation system. The roaming wave is a passenger, bonding points are intersections, residential waves are vehicles, strong axes $2 \mathrm{~A}^{2}$ are highways.

The roaming wave decides the next direction at the point of bonding between nucleons where the strong interactions take place. It chooses a vehicle randomly to make a turn or move straight.

Since wave $2 * 2$ interacts with the inner wave 2 of $\mathrm{A}^{2}$, it helps passing wave moves straight when crossing the interacting point of $2 \mathrm{~A}^{2}$. Wave $2 * 3$ interacts mainly with wave 5 of $\mathrm{A}^{2}$. It helps the passing wave make directional changes or keep straight when crossing the interaction point of $2 \mathrm{~A}^{2}$. Wave $2+3$ helps the passing wave make directional changes and coupling with neighboring wave $2 * 2$.

In a nucleus other than the hydrogen nucleus, $\mathrm{A}(2 * 3 * 5)$ changes to $\mathrm{B}(2 * 2 * 4)$ to resonant with the other nuclei properly by improving resonance with the inner wave 2 of $\mathrm{A}^{2}$. The wave B can be charged $2 * 2 * 4$ or neutral $2 * 2 * 2 * 2$.

\subsection{Configuration of Proton}

If we use the electron as the unit mass, we can see that the mass of the proton is:

1836.15267(e) $=2 \mathrm{~A}^{2}+\mathrm{A}+2 * 3+0.15267$

The additional mass 0.15267 is result of interactions in nucleus.

The structure $2 \mathrm{~A}^{2}$ strong interaction:

$137 / 900=0.152222$

The weak interaction with charge vectors vertical to eight faces of octahedron structure is:

$8 /(137 * 137)=0.000426$

Wave $2 * 3$ weakly interacts with A $(2 * 3 * 5) .5$ is not direct energy and it has a factor of $1 / 2$. The self-dissonance of the weak interaction wave of $2 * 3 * 5$ is:

$2.5 /(137 * 137 * 2 * 3)=0.000022$

This number matches exactly to the known Proton interactive mass 0.15267 :

$0.152222+0.000426+0.000022=0.15267$

\subsection{Configuration of Neutron}

Using electron mass as a unit mass, we can calculate the mass of a proton to be: 
$1838.68365987=2 \mathrm{~A}^{2}+\mathrm{A}+2 * 3+2.5+0.15267+0.030987$

The Neutron is a Proton plus 2.5 mass waves. The 2.5 wave has a strong interaction with two $\mathrm{A}^{2}$ structure with bonding energy of:

$137 /(900 * 2.5 * 2)=0.030444$

For dissonant weak interactions between 2.5 and $2 * 3$, 2.5 needs a factor of 2 to become $5.2+3=5$, but it is not direct energy. It has factor of $1 / 2$ :

$\mathrm{P}=2 * 2.5$

$\mathrm{Q}=1 / 2$

$\mathrm{P} / \mathrm{Q}=(2 * 2.5 /(1 / 2))=10$

$(\mathrm{P} / \mathrm{Q}) /(137 * 137)=0.0005328$

Weak interaction between 2.5 and $2 * 3 * 5$ is related to the opposite charge. 2.5 needs factor 2 and 3 to interact:

$1 /(137 * 137 * 2 * 3)=0.00001$

$0.030444+0.0005328+0.00001=0.030987$

The number matches exactly with the known Neutron mass.

\subsection{Coupling of Proton and Neutron}

Proton:

$2 \mathrm{~A}^{2}+\mathrm{A}+2 * 3+137 / 900+8 /(137 * 137)+(5 / 2) /(137 * 137 * 2 * 3)$

Neutron:

Proton $+137 /(900 * 2.5 * 2)+10 /(137 * 137)+1 /(137 * 137 * 6)$

When a proton and a neutron form a nucleus, one of the axes A from proton bonds with an A axis from neutron introduce a new bonding force of $137 / 900$. Wave $2 * 3$ becomes $2 * 2$ to resonant with two nodes.

The wave 2.5 of neutron becomes 2 to resonant with the other $2 * 2$ waves. The dissonant weak interaction of 2.5 with $2 * 3$ no longer exists since $2 * 3$ wave changed to $2 * 2$.

Wave $2 * 2$ weakly interacts with A $(2 * 3 * 5) .5$ is not direct energy and it has factor of $1 / 2$. The self-dissonance weak interaction wave of $2 * 3 * 5$ is:

$5 /(137 * 137 * 2 * 2 * 3)=0.000022$

A transformed proton and neutron has lower energy in the ${ }^{2} \mathrm{H}$ nucleus.

Proton:

$2 \mathrm{~A}^{2}+\mathrm{A}+2 * 2+137 / 900+8 /(137 * 137)+137 /(900 * 3 * 2)+5 /(137 * 137 * 2 * 2 * 3)=1834.15267$

Neutron:

$2 \mathrm{~A}^{2}+\mathrm{A}+2 * 2+2+137 / 900+8 /(137 * 137)+5 /(137 * 137 * 2 * 2 * 3)+1 /(137 * 137 * 6)=1836.17805$

Strong interaction on the bonding point:

$1 * 137 / 900$

Total: 3670.48294

It matches exactly to the known value: 3670.48294 of ${ }^{2} \mathrm{H}$ mass.

\subsection{Two Neutrons and One Proton}

Since there are two neutrons, two negative waves 3 are shared among them. Proton only contains three axes:

$3 *\left(2 \mathrm{~A}^{2}+\mathrm{A}\right)+2 * 3=5496$

Two energy 3 waves strongly interact with three nucleons:

$2 * 3 * 137 / 900=0.9133333$

The 2.5 wave in neutron is missing; the neutron bonding remains, as there are one more neutrons than protons.

The energy is reduced to one quarter of neutron bonding:

$137 /(900 * 5 * 2 * 2)=0.0076111$

The weak interaction on eight faces of octahedron structure:

$8 /(137 * 137)=0.000426$

The weak interaction of two wave 3 :

$2 /(137 * 137)=0.000107$

The total mass: 5496.9215

It matches the experimental data of ${ }^{3} \mathrm{H}$ mass: 5497.9215 


\subsection{Two Protons and One Neutron}

Since there is single neutron, only has single wave 5:

$2 *\left(2 A^{2}+A\right)+\left(2 A^{2}+A+5\right)=5495$

$A^{2}$ aggregates with $A$ during interaction $\left(A^{2}+A=930\right)$ as charged protons dominate the nucleus. Three nucleons strongly interact via two bonding points. But each bonding point has half the energy since the nucleons can rotate.

$2 * 137 /(930 * 2)=0.147312$

Dissonance wave of 5:

$5 * 137 / 930=0.736559$

Additional interactions are between 930, 30 and 5:

$137 /(930 * 30 * 5)=0.001$

The total mass: 5495.8851 matches the experimental data.

\subsection{Two Neutrons and Two Protons}

Since there are two neutrons, two positive waves 3 are shared among them. The charged axis A changes to B (2 $* 2 * 4$ ) to interact with the central waves of $2 \mathrm{~A}^{2}$. Wave $2 * 3$ facilitates passing wave's direction changes. Energy formula becomes:

$$
4 *\left(2 \mathrm{~A}^{2}+\mathrm{B}+2 * 3\right)+2 * 3=7294
$$

Four nucleons strongly interact via four bonding points. But each bonding point has half energy since the nucleons can rotate. $A^{2}$ aggregates with $B$ during interaction $\left(A^{2}+B=916\right)$ as charged protons dominate the nucleus:

$$
4 * 137 /((900+2 * 2 * 4) * 2)=0.29912664
$$

Weak interaction:

$4 / 137 * 137=0.0002131173$

The total mass: 7294.29933975 matches the experimental data: 7294.299

\subsection{Lithium (Figure 25)}

Wave $2 * 2$ helps straighten movement and wave $2 * 3$ helps with turning. Wave $2+3$ as shape effects for tilted $2 * 3$ shape (Figure 25):

$$
6 *\left(2 A^{2}+B+2 * 2+2 * 3\right)+2+3=10961
$$

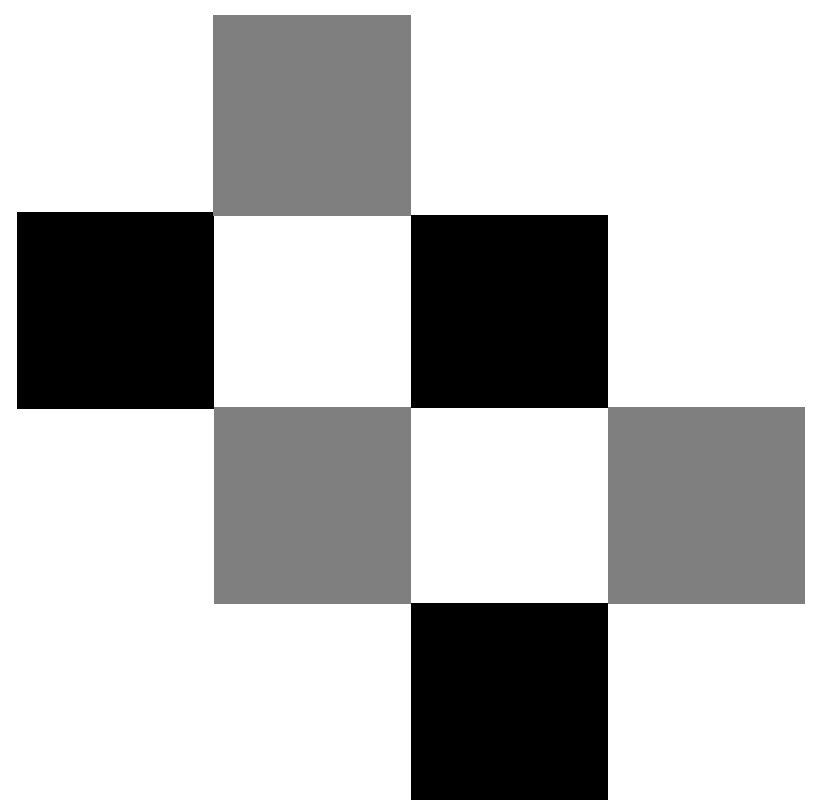

Figure 25. ${ }^{6}$ Lithium. 
Three nucleons strongly interact via seven bonding points, while two of them have half energy. $\mathrm{A}^{2}$ aggregates with $\mathrm{B}$ during interaction $\left(\mathrm{A}^{2}+\mathrm{B}=916\right)$ as charged protons dominate the nucleus:

$6 * 137 /(916)=0.89738$

Weak interaction:

$6 /(137 * 137)=0.00032$

Matches the experimental data: 10961.8977

\section{8. ${ }^{9}$ Beryllium}

Two pairs of proton lower $2 * 3$ wave to $2 * 2$ from Li. Its symmetrical shape makes two $2 * 2$ waves as shape (Figure 26) effects:

$9 *\left(2 A^{2}+B+2 * 2+2 * 2\right)+2 * 2+2 * 2=16424$

Strong interaction:

$9 * 137 /(926 * 5)=0.2663$

Closely matches the experimental data: 16424.2504

\section{9. ${ }^{10}$ Boron}

Two pairs of proton lower $2 * 3$ wave to $2 * 2$ from Li. The tilted 2, 3 and 4 nucleon lines can explain wave $3+$ 4 as shape (Figure 27) effects:

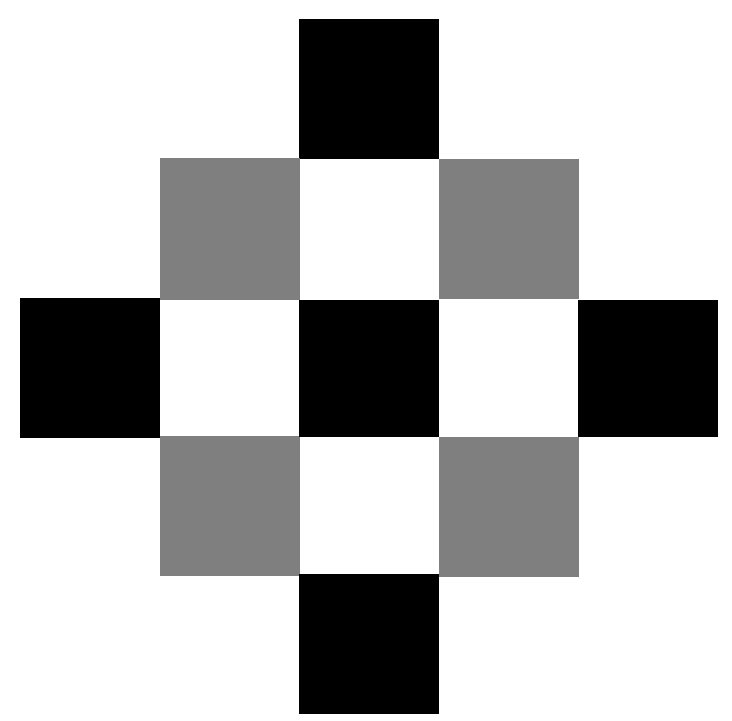

Figure 26. ${ }^{9}$ Beryllium.

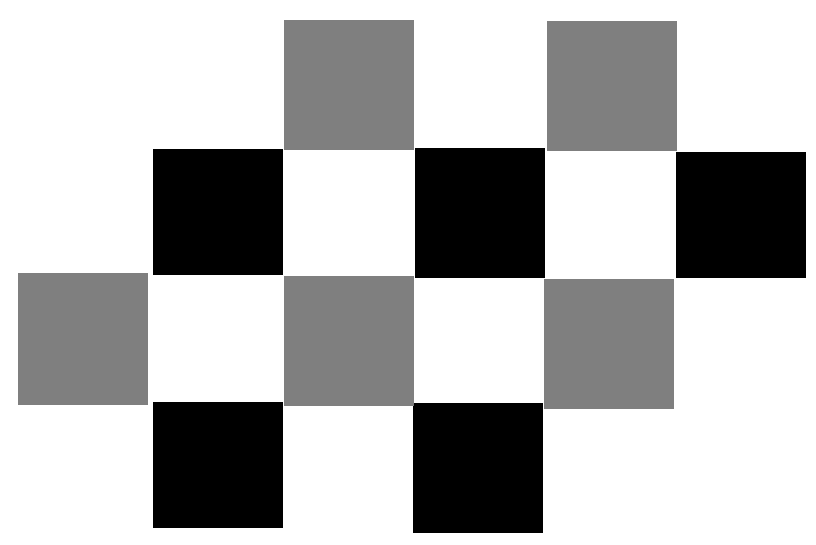

Figure 27. ${ }^{10}$ Boron. 
$10 *\left(2 \mathrm{~A}^{2}+\mathrm{B}+2 * 2+2 * 2\right)+3+4=18247$

Three nucleons strongly interact via thirteen bonding points each shared by four parties. $A^{2}$ does not aggregate with $\mathrm{B}$ during interaction similar to Beryllium case:

$13 * 137 /(900 * 4)=0.456722$

Additional unexplained strong interactions will be ignored:

$137 /(900 * 3 * 5)=0.010148$

Closely matches the experimental data: 18247.466754

\subsection{0. ${ }^{12}$ Carbon}

Three pairs of protons without an internal proton (proton is not paired with external proton) reduce $2 * 2+2 * 2$ to $2 * 3$. Only two or one protons in diagonal lines, they prefer interactive wave of $2 * 2$ as square shape (Figure 28) effects:

$12 *\left(2 \mathrm{~A}^{2}+\mathrm{B}+2 * 3\right)+2 * 2=21868$

The nucleons strongly interact via sixteen bonding points in the center with $\mathrm{A}^{2}$ and $2 * 2$ :

$16 * 137 /(900 * 2 * 2)=0.60889$

Dissonant wave bonding:

$2 * 137 /(900 * 2 * 3)=0.05074$

Matches the experimental data: 21868.66

\subsection{Oxygen}

The twelve outer nucleons have wave $2+3$ while four corner nucleons (Figure 29) have wave 2 * 3 :

$4 *\left(2 \mathrm{~A}^{2}+\mathrm{B}+2 * 3\right)+12 *\left(2 \mathrm{~A}^{2}+\mathrm{B}+2+3\right)+2 * 2=29148$

The nucleons strongly interact via 24 bonding points in the center. $\mathrm{A}^{2}$ aggregates with $\mathrm{B}$ during interaction. Twenty four bonding points interact with $2 * 2$ :

$24 * 137 /(900 * 2 * 2)=0.7611111$

Dissonance of $2 * 3$ and $(2+3)$ :

$(2 * 3) * 137 /(900 *(2+3))=0.182666$

Interaction of $\mathrm{B}$ and $(2+3)$ :

$137 /(900 *(2+3) * 16)=0.0019$

This matches the experimental data: 29148.9457

\subsection{2. ${ }^{27}$ Aluminium}

The existence of internal proton prefers wave $2+3$. Shared wave $2 * 2$ as effects for the shape (Figure 30 ):

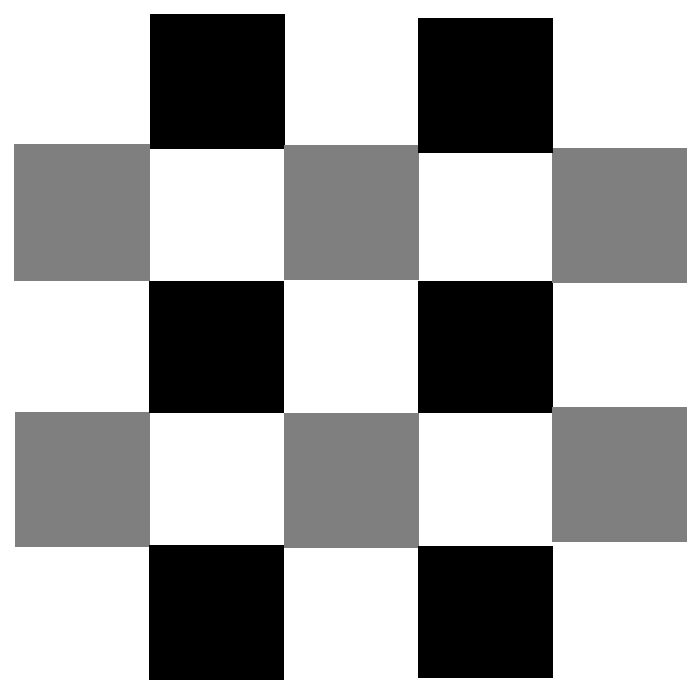

Figure 28. ${ }^{12}$ Carbon. 

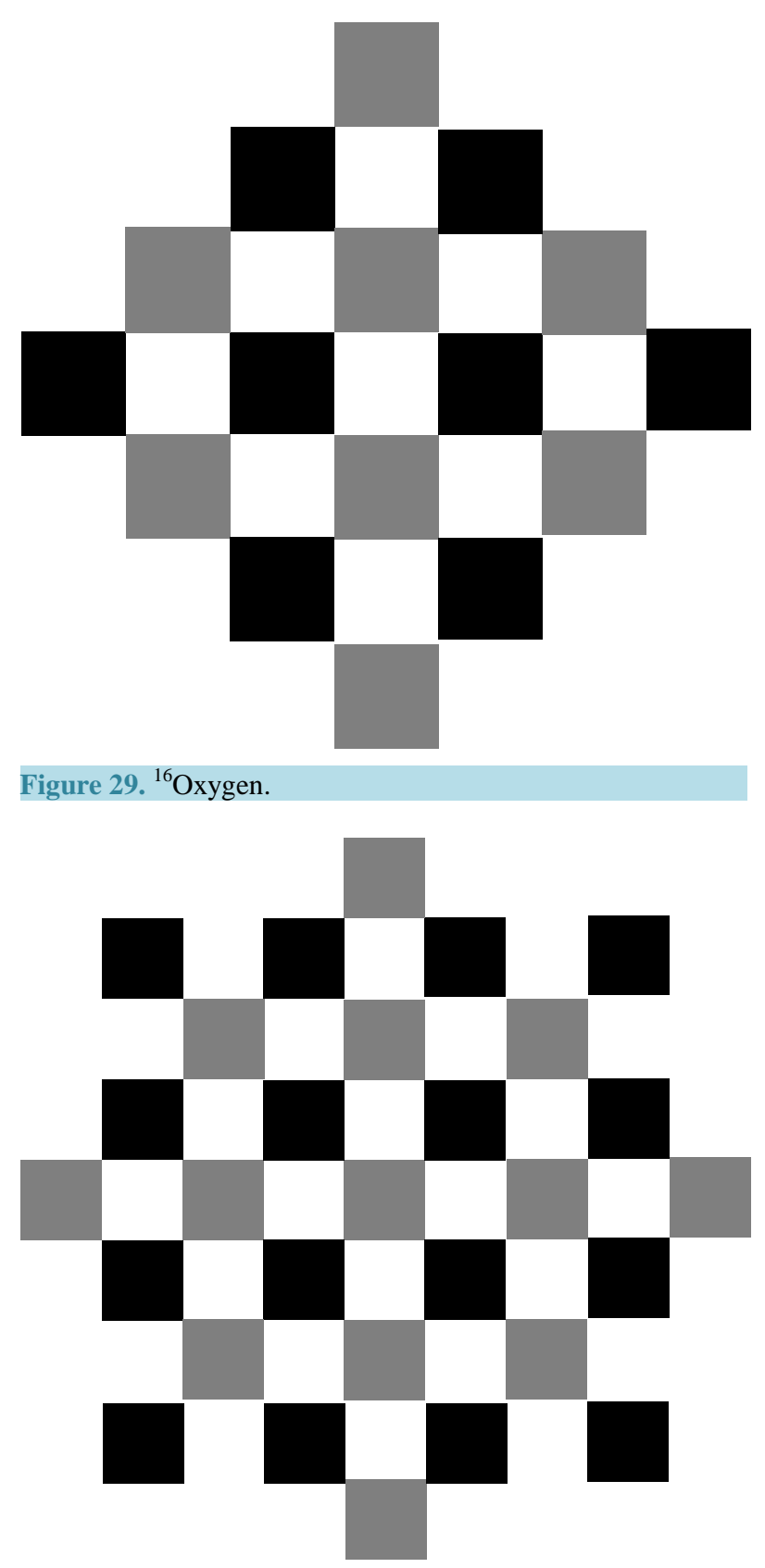

Figure 30. ${ }^{27}$ Aluminium.

$27 *\left(2 \mathrm{~A}^{2}+\mathrm{B}+2+3\right)+2 * 2=29148$

The nucleons strongly interact via 44 bonding points. $\mathrm{A}^{2}$ does not aggregate with $\mathrm{B}$ during interaction as more neutrons are involved:

$44 * 137 /(900 * 2 * 2 *(2+3))=0.33489$

It matches the experimental data: 49171.33606

\subsection{3. ${ }^{28}$ Silicon}

The existence of internal proton in ${ }^{28}$ Silicon (Figure 31) prefers wave $2+3$ for external protons and neutron pairs. The six internal neutrons and two internal protons prefer $2 * 2$ :

$20 *\left(2 A^{2}+B+2+3\right)+8 *\left(2 A^{2}+B+2 * 2\right)+2 * 2=50984$ 


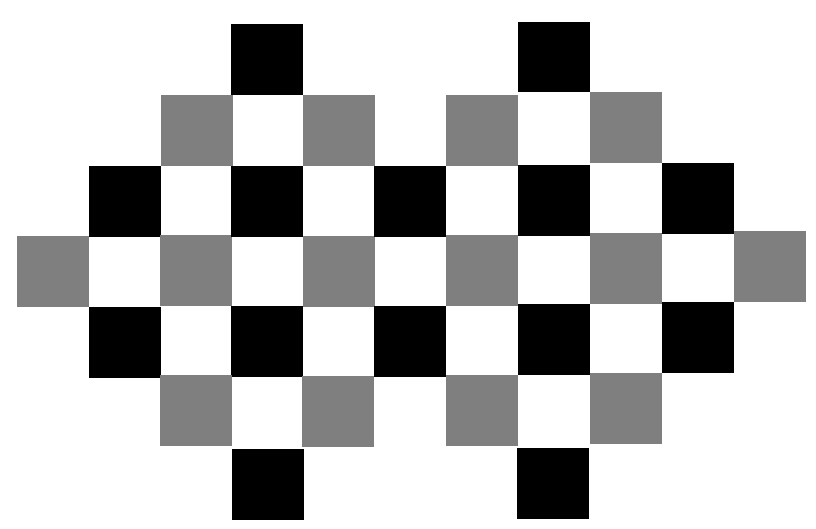

Figure 31. ${ }^{28}$ Silicon.

The nucleons strongly interact via 42 bonding points. $\mathrm{A}^{2}$ does not aggregate with $\mathrm{B}$ during interaction, $\mathrm{E}=2$ * $2 * 2$ instead of $2 * 2 *(2+3)$ since wave $2 * 2$ resonant with shared wave $2 * 2$ :

$42 * 137 /(900 * 2 * 2 * 2)=0.7992$

$(2+3)$ strong interaction:

$2 * 137 /(900 * 2 * 2 *(2+3))=0.0152$

Matches the experimental data: 50984.8144

\subsection{Summary}

Omitting the axes structural components, $\mathrm{A}^{2}$ and $\mathrm{B}$, the main structural components are (Table 3).

\section{Discussion}

The energy calculations are studied for the lower mass elements. As elements become heavier, the strong interaction results become simpler and less accurate as the paper ignores smaller factors mainly related to weak interactions.

The structures of the nuclei are pretty certain for each isotopes, as it is impossible to find better alternatives for Carbon (Figure 32) as follows:

It is pretty obvious that there is no way to make ${ }^{11}$ Carbon (Figure 33) symmetrical:

In general, the symmetrical nuclei provide better circling passes for the roaming energy wave. Inner nucleons in proton/neutron pair allows roaming wave goes straight and it prefers wave $2 * 2$. The external nucleons prefer $2 * 3$ or $2+3$ as the roaming wave has to change directions. The heavy mass elements prefer $2 * 2 * 4$ (B) charge axes. For neutron, the B axes are neutral $(2 * 2 * 2 * 2)$.

\subsection{Nuclear Magnetic Resonance Sensitivity}

${ }^{1} \mathrm{H}$ (Figure 34) is the nucleus most sensitive to NMR signal. Proton NMR produces narrow chemical shift with sharp signals. Fast acquisition of quantitative results (peak integrals in stoichiometric ratio) is possible due to short relaxation time. The ${ }^{1} \mathrm{H}$ signal has been the sole diagnostic nucleus used for clinical magnetic resonance imaging.

${ }^{3} \mathrm{He}$ (Figure 34), is very sensitive to NMR. There is a very low percentage in natural helium, and subsequently has to be purified from $4 \mathrm{He}$.

${ }^{19} \mathrm{~F}$ (Figure 34), relatively commonly measured. Sensitive, yields sharp signals, has wide chemical shift.

The energy calculations are studied for the lower mass elements. As elements become heavier, the strong interaction results become simpler and less accurate as the paper ignores smaller factors mainly related to weak interactions.

Not NMR sensitive (Figure 35):

The non-metal nuclei with stretched out configuration are more sensitive to NMR. Following is predicted configuration of ${ }^{29} \mathrm{Si}$ (Figure 36):

Alternatively (Figure 37), it can have the following configuration: 


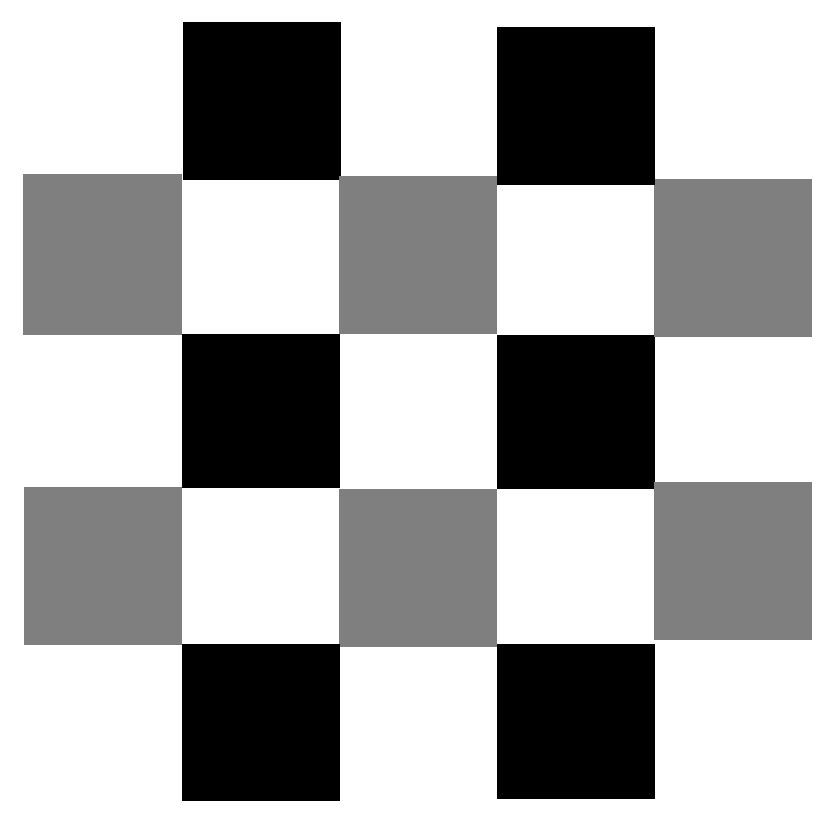

Figure 32. ${ }^{12}$ Carbon.

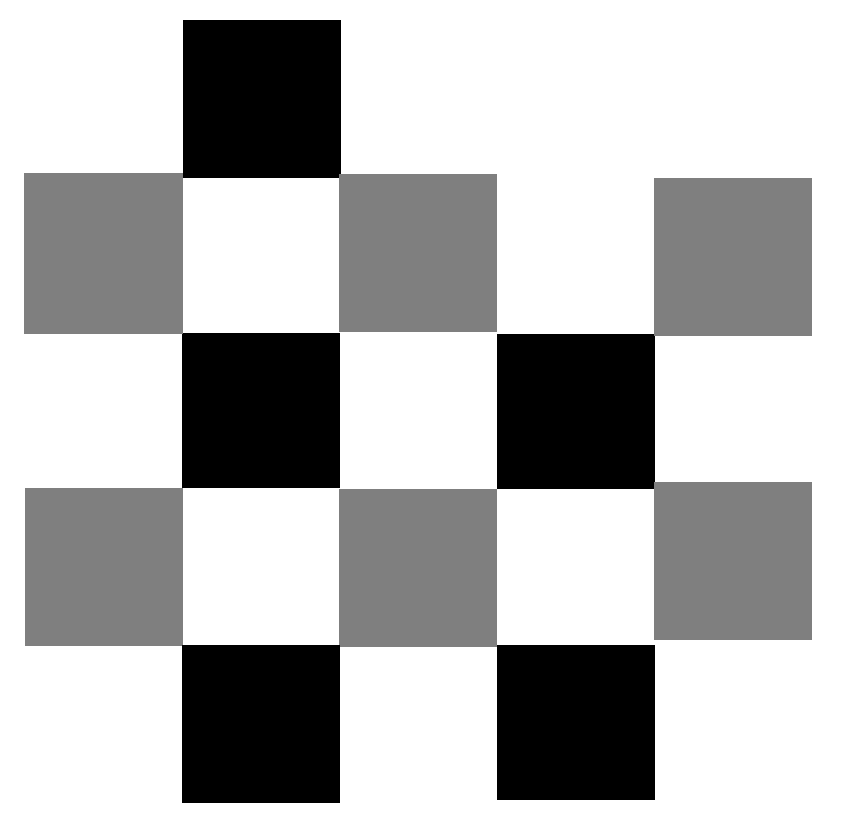

Figure 33. ${ }^{11}$ Carbon.

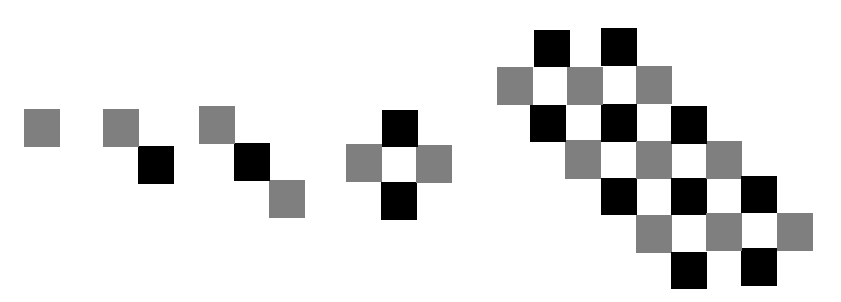

Figure 34. NMR Sensitive.

Knowing that ${ }^{29} \mathrm{Si}$ is a lot NMR sensitive than ${ }^{28} \mathrm{Si}$, it has to be configured as predicted structure with the following structural formula: 

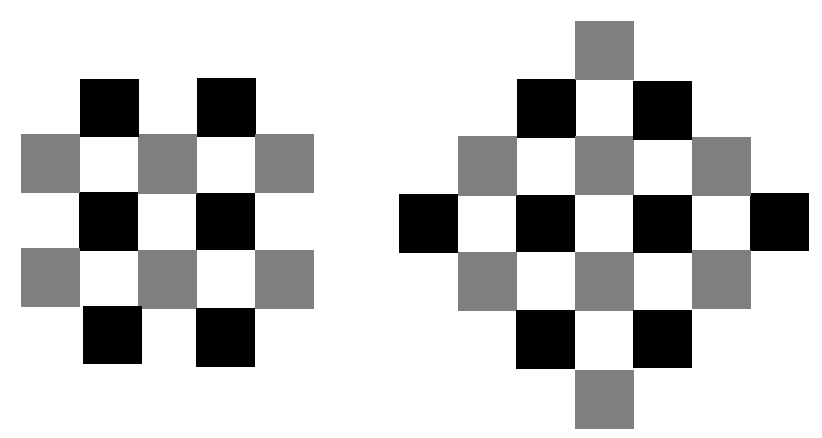

Figure 35. Not nmr sensitive.

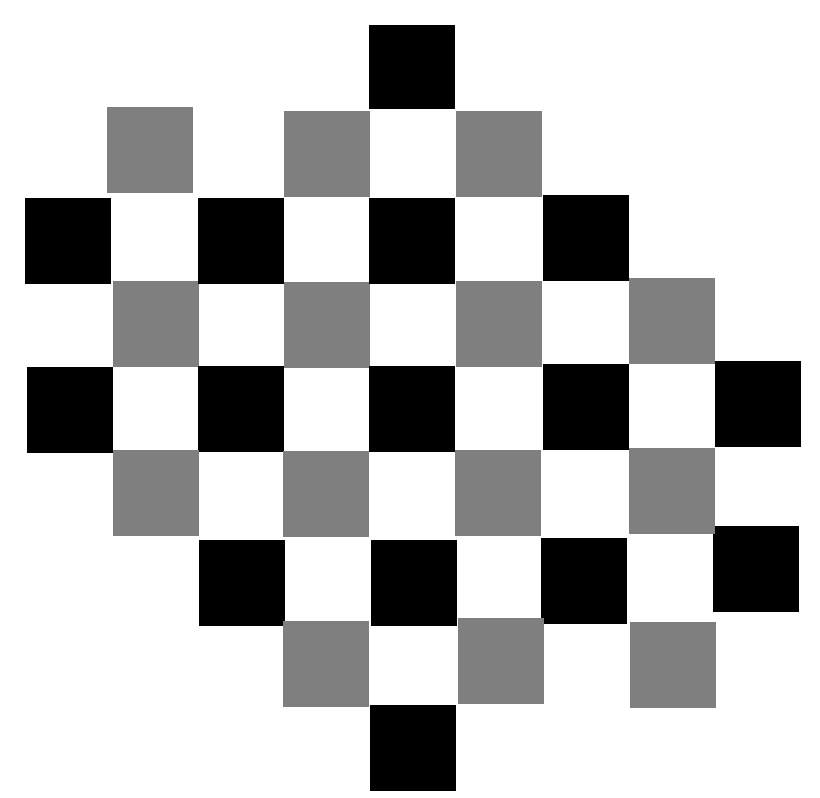

Figure 36. ${ }^{29}$ Si predicted lattice confirmed by NMR.

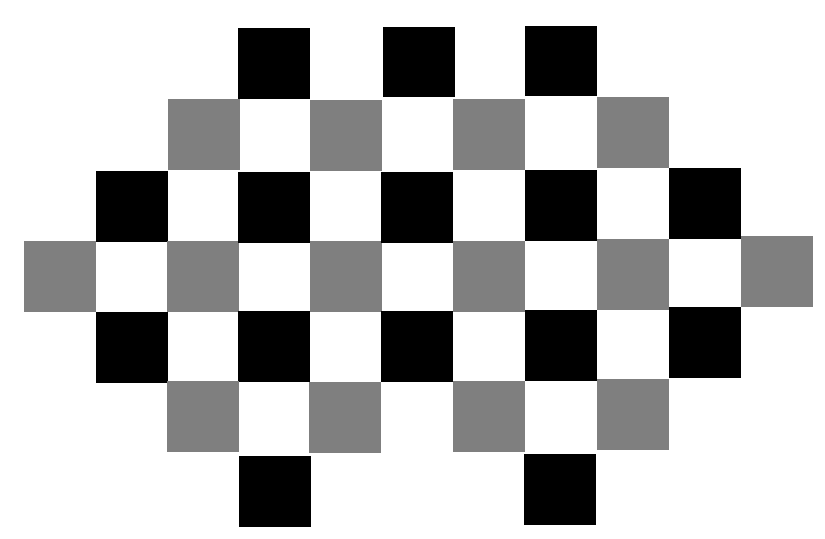

Figure 37. Alternative lattice not NMR sensitive.

$22 *\left(2 A^{2}+B+2+3\right)+9 *\left(2 A^{2}+B+2 * 2\right)+2 * 3$

\subsection{Mass Calculation Confirmation}

Once the structure of a nucleus is decided, energy of the nucleus can be analyzed. The accurate calculation result from simplified BCS formalism can be used to confirm the predicted nuclear structure. 


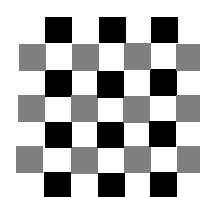

$2 * 3$

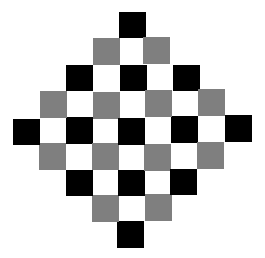

$3 * 3$

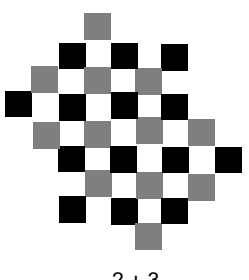

Figure 38. Lattice confirm by calculation.

Table 3. Mass formula.

\begin{tabular}{|c|c|c|c|}
\hline Element & $\mathrm{Z}$ & NMR Properties & Mass in Atomic Unit (u) \\
\hline $\mathrm{He}$ & 2 & $(Z+N)(2 * 3)+2$ & 1823.574845 \\
\hline $\mathrm{Li}$ & 3 & $(\mathrm{Z}+\mathrm{N})(2 * 3)+2 * 3$ & 1826.979364 \\
\hline $\mathrm{Be}$ & 4 & $(\mathrm{Z}+\mathrm{N})(2 * 2+2 * 3)+2+3$ & 1824.911461 \\
\hline B & 5 & $(\mathrm{Z}+\mathrm{N})(2 * 2+2 * 2)+3+4$ & 1823.976003 \\
\hline C & 6 & $(\mathrm{Z}+\mathrm{N})(2 * 3)+2 * 2$ & 1822.388485 \\
\hline N (15) & 7 & $15(2 * 3)+2 * 3$ & 1822.788739 \\
\hline $\mathrm{O}$ & 8 & $4(2 * 3)+12(2+3)+2 * 2$ & 1821.809104 \\
\hline $\mathrm{F}$ & 9 & $(\mathrm{Z}+\mathrm{N})(2 * 3)+2 * 2$ & 1822.261603 \\
\hline $\mathrm{Ne}$ & 10 & $(\mathrm{Z}+\mathrm{N})(2+3)+2 * 3$ & 1821.699449 \\
\hline $\mathrm{Na}$ & 11 & $17(2+3)+6(2 * 3)+3+4$ & 1821.599378 \\
\hline $\mathrm{Mg}$ & 12 & $(Z+N)(2+3)+2 * 3$ & 1821.252346 \\
\hline $\mathrm{Al}$ & 13 & $(Z+N)(2+3)+2 * 2$ & 1821.160595 \\
\hline $\mathrm{Si}$ & 14 & $20(2+3)+8(2 * 2)+2 * 2$ & 1820.886329 \\
\hline $\mathrm{P}$ & 15 & $20(2+3)+11(2 * 2)+2 * 3$ & 1820.861722 \\
\hline S & 16 & $13(2 * 2)+19(2+3)+2 * 3$ & 1820.828752 \\
\hline $\mathrm{Fe}(56)$ & 26 & $45(2 * 2)+11(2+3)+2 * 3$ & 1820.306312 \\
\hline $\mathrm{Fe}(57)$ & 26 & $45(2 * 2)+14(2+3)+2 * 3$ & 1820.366212 \\
\hline
\end{tabular}

${ }^{24} \mathrm{Mg},{ }^{25} \mathrm{Mg}$ and ${ }^{26} \mathrm{Mg}$ (Figure 38) have following mass calculation:

${ }^{24} \mathrm{Mg}: 24 *\left(2 \mathrm{~A}^{2}+\mathrm{B}+2+3\right)+2 * 3$

${ }^{25} \mathrm{Mg}: 25 *\left(2 \mathrm{~A}^{2}+\mathrm{B}+2+3\right)+3 * 3+25 * 137 /(900 * 10)$

${ }^{26} \mathrm{Mg}: 26 *\left(2 \mathrm{~A}^{2}+\mathrm{B}+2+3\right)+2+3$

\section{Conclusion}

A better understanding on nuclear structure is essential for future nuclear research. Unfortunately, detailed nuclear configuration cannot be observed visually. Since a nucleus is composed of neutron and proton, the nucleus is considered to be a product of many body interactions. The existing many body theories may be at a dead end and there have been no major developments since then. A new Unified Field Theory created more than ten years ago predicted detailed configuration of nuclei recently. The predicted configurations of nuclei can make nuclear "clear" and it narrows down the possible variations of nuclear structures. The proposed trial and error method not only can study nuclear configuration for lower mass nuclei but also can study configuration of any isotope of 
known mass. We anticipate our essay to be a starting point for more sophisticated studies on nuclear physics. For example, structure for each isotope can be discovered with help of the methods demonstrated in the paper.

\section{References}

[1] Berbiche, A., Fellah, M. and Allal, N.H. (2014) Number-Projected Isovector Neutron-Proton Pairing Effect in Odd Mass Nuclei. Journal of Theoretical and Applied Physics, 8, 118.

[2] Goodman, A. (1979) Hartree-Fock-Bogoliubov Theory. Advances in Nuclear Physics, 11, 263-366.

[3] Civitarese, O. and Reboiro, M. (1997) Proton-Neutron Pairing Effects in Medium and Heavy Mass Nuclei. Physical Review C, 56, 1179-1182. http://dx.doi.org/10.1103/PhysRevC.56.1179

[4] Civitarese, O., Reboiro, M. and Vogel, P. (1997) Neutron-Proton Pairing in the BCS Approach. Physical Review C, 56, 1840-1843. http://dx.doi.org/10.1103/PhysRevC.56.1840

[5] Vogel, P. (1998) Neutron-Proton Pairing. Czechoslovak Journal of Physics, 48, 269-276. http://dx.doi.org/10.1023/A:1021292614670

[6] Simkovic, F., Moustakidis, C.C., Pacearescu, L. and Faessler, A. (2003) Proton-Neutron Pairing in the Deformed BCS Approach. Physical Review C, 68, Article ID: 054319. http://dx.doi.org/10.1103/PhysRevC.68.054319

[7] Mokhtari, D., Ami, I., Fellah, M. and Allal, N.H. (2008) Neutron-Proton Isovector Pairing Effect on the Nuclear Moment of Inertia. International Journal of Modern Physics E, 17, 655-667. http://dx.doi.org/10.1142/S0218301308010064

[8] Fellah, M., Allal, N.H., Belabbas, M., Oudih, M.R. and Benhamouda, N. (2007) Temperature-Dependent Isovector Pairing Gap Equations Using a Path Integral Approach. Physical Review C, 76, Article ID: 047306. http://dx.doi.org/10.1103/PhysRevC.76.047306

[9] Skrypnykm, T. (2012) Rational R-Matrices, Higher Rank Lie Algebras and Integrable Proton-Neutron BCS Models. Nuclear Physics B, 863, 435-469.

[10] Cao, Z.L. and Cao, H.G. (2014) Unified Field Theory and Topology of Nuclei. International Journal of Physics, 2, 1522. http://dx.doi.org/10.12691/ijp-2-1-4

[11] Cao, Z.L., Cao, H.G. and Qiang, W. (2015) Unified Field Theory and Topology of Atom. American Journal of Modern Physics, Special Issue, 4, 1-7.

[12] Cao, Z.L. and Cao, H.G. (2014) Cubic Atom and Crystal Structures. International Journal of Physics, 2, 277-281. http://dx.doi.org/10.12691/ijp-2-6-11

[13] Cao, Z.L., Cao, H.G. and Qiang, W. (2015) Theory of Everything. Frontiers of Astronomy, Astrophysics and Cosmology, 1, 31-36.

[14] Cao, Z.L. and Cao, H.G. (2013) Unified Field Theory and the Configuration of Particles. International Journal of Physics, 1, 151-161.

[15] Cao, Z.L. and Cao, H.G. (2014) Unified Field Theory and Foundation of Physics. International Journal of Physics, 2 , 158-164. http://dx.doi.org/10.12691/ijp-2-5-5

[16] Cao, Z.L. and Cao, H.G. (2013) Unified Field Theory. American Journal of Modern Physics, 2, 292-298. http://dx.doi.org/10.11648/j.ajmp.20130206.14

[17] Cao, Z.L. and Cao, H.G. (2013) Unified Field Theory and the Hierarchical Universe. International Journal of Physics, 1, 162-170.

[18] Cao, H.G. and Cao, Z.L. (2013) Drifting Clock and Lunar Cycle. International Journal of Physics, 1, 121-127.

[19] Cao, Z.L. and Cao, H.G. (2013) Non-Scattering Photon Electron Interaction. Physics and Materials Chemistry, 1, 9-12.

[20] Cao, Z.L. and Cao, H.G. (2013) SR Equations without Constant One-Way Speed of Light. International Journal of Physics, 1, 106-109.

[21] Myers, E.G., Wagner, A., Kracke, H. and Wesson B.A. (2015) Atomic Masses of Tritium and Helium-3. Physical Review Letters, 114, Article ID: 013003.

[22] Chekhovich, E.A., Hopkinson, M., Skolnick, M.S. and Tartakovskii, A.I. (2015) Suppression of Nuclear Spin Bath Fluctuations in Self-Assembled Quantum Dots Induced by Inhomogeneous Strain. Nature Communications, 6, 6348. http://dx.doi.org/10.1038/ncomms7348

[23] Dong, Y., Dong, L., Gong, M. and Pu, H. (2015) Dynamical Phases in Quenched Spin-Orbit-Coupled Degenerate Fermi Gas. Nature Communications, 6, 6103. http://dx.doi.org/10.1038/ncomms7103

[24] Koizumi, H., Okazaki, A., Ghantous, M.A. and Tachiki, M. (2014) Supercurrent Flow through the Network of Spin-Vortices in Cuprates. Journal of Superconductivity and Novel Magnetism, 27, 2435-2446. 
http://dx.doi.org/10.1007/s10948-014-2626-9

[25] Tan, Z.B., Cox, D., Nieminen, T., Lähteenmäki, P., Golubev, D., Lesovik, G.B. and Hakonen P.J. (2015) Cooper Pair Splitting by Means of Graphene Quantum Dots. Physical Review Letters, 114, Article ID: 096602.

[26] Tan, D., Weber S.J., Siddiqi, I., Mølmer, K. and Murch, K.W. (2015) Prediction and Retrodiction for a Continuously Monitored Superconducting Qubit. Physical Review Letters, 114, Article ID: 090403.

[27] Murmann, S., Bergschneider, A., Klinkhamer, V.M., Zürn, G., Lompe, T. and Jochim, S. (2015) Two Fermions in a Double Well: Exploring a Fundamental Building Block of the Hubbard Model. Physical Review Letters, 114, Article ID: 080402.

[28] Giraud, O., Braun, D., Baguette, D., Bastin, T. and Martin, J. (2015) Tensor Representation of Spin States. Physical Review Letters, 114, Article ID: 080401.

[29] Valderrama, M.P. and Phillips, D.R. (2015) Power Counting of Contact-Range Currents in Effective Field Theory. Physical Review Letters, 114, Article ID: 082502.

[30] Phillips, D.R., Samart, D. and Schat, C. (2015) Parity-Violating Nucleon-Nucleon Force in the 1/Nc Expansion. Physical Review Letters, 114, Article ID: 062301

[31] Stetcu, I., Bertulani, C.A., Bulgac, A., Magierski, P. and Roche K.J. (2015) Relativistic Coulomb Excitation within the Time Dependent Super Fluid Local Density Approximation. Physical Review Letters, 114, Article ID: 012701

[32] Borsanyi, S., Durr, S., Fodor, Z., Hoelbling, C., Katz, S.D., Krieg, S., et al. (2015) Ab Initio Calculation of the Neutron-Proton Mass Difference. Science, 347, 1452-1455.

[33] Freedman, R. and Young, H. (2004) Sears and Zemanskey’s University Physics with Modern Physics. 11th Edition, Benjamin-Cummings Pub Co, San Francisco, 1633-1634. 